\title{
A SIGHT FOR SORE EYES The Return of True Color to Geostationary Satellites
}

\author{
by Steven D. Miller, Timothy L. Schmit, Curtis J. Seaman, Daniel T. Lindsey, \\ Mathew M. Gunshor, Richard A. Kohrs, Yasuhiko Sumida, and Donald Hillger
}

\begin{abstract}
Japan's Himawari-8 Advanced Himawari Imager enables use of the first true-color imagery from the geostationary orbit in nearly half a century and previews advanced capabilities of the next-generation international constellation.
\end{abstract}

T he old adage goes that "a picture is worth a thousand words." When it comes to pictures taken of Earth from the vantage point of space, these words ring especially true. Iconic examples include the "Earth rise" over a lunar foreground, captured by the crew of Apollo 8 in December 1968, and the "Blue Marble" taken by the crew of Apollo 17

AfFiliations: Miller AND SeAman-Cooperative Institute for Research in the Atmosphere, Colorado State University, Ft. Collins, Colorado; ScHMIT-NOAA/Center for Satellite Applications and Research, Advanced Satellite Products Branch, Madison, Wisconsin; LINDSEY AND HILlger-NOAA/Center for Satellite Applications and Research, Regional and Mesoscale Meteorology Branch, Ft. Collins, Colorado; GUNSHOR AND KoHRSCooperative Institute for Meteorological Satellite Studies, Space Science and Engineering Center, University of Wisconsin-Madison, Madison, Wisconsin; SUMIDA-Meteorological Satellite Center, Japan Meteorological Agency, Tokyo, Japan

CORRESPONDING AUTHOR: Steven D. Miller, Ph.D., Cooperative Institute for Research in the Atmosphere, Colorado State University, Foothills Campus, 1375 Campus Delivery, Ft. Collins, CO 80523

E-mail: steven.miller@colostate.edu

The abstract for this article can be found in this issue, following the table of contents.

DOI:10.1175/BAMS-D-15-00154.1

A supplement to this article is available online (10.1175/BAMS-D-15-00154.2)

In final form 18 February 2016

(C2016 American Meteorological Society in December 1972. They reveal to our eyes a strikingly colorful, dynamic, and borderless world-one whose form and function as a kind of living, breathing organism can only be realized from the holistic view. From afar, the Blue Marble conveys a peaceful and uniting message of "home" to a world embroiled in unrest and division.

Practically speaking, imaging radiometers provide a unique dual perspective on Earth's complexity and connectivity. In the most basic sense, the "eyes" of satellite radiometers collect energy in the form of photons impacting a detector-the more energy received, the higher the digital count value. These counts are converted into Earth-located measurements of reflected sunlight and thermal emission. This quantitative information resides at the spatial granularity of picture elements, or pixels, collected via scan patterns that vary with satellite orbital configuration and instrumentation. When considered at the pixel level, the data offer an ability to quantify the properties of the surface and atmosphere at a discrete viewing location. When displayed as georeferenced (or mapped) imagery, we are able to visualize how each pixel's information fits within the spatial context of the whole scene. These two forms of information content-quantitative products and qualitative context-are fully complementary and inseparable attributes of satellite data.

Satellite imagery comes in many forms-from simple, single-band images for a specific spectral 
range to more sophisticated (and higher information content) multispectral-band combinations rendered via red-green-blue "false color" composite techniques (d'Entremont and Thomason 1987). Among the latter, the special case of "true color" is perhaps the single most familiar and visually intuitive form of multispectral satellite imagery (Miller et al. 2012). True to its name, true color combines appropriately scaled red-, green-, and blue-band reflectance values to form imagery that approximates the response of normal human photopic (daytime) vision. In essence, true color mimics what we see with our own eyes, producing an image reminiscent of color photography.

When it comes to daytime interpretation of surface and atmospheric properties, true-color imagery offers distinct and practical advantages. With true color, the oceans appear blue, shoals and coastal waters are turquoise, the forests are green, and the deserts are tan. In panchromatic visible imagery, the millions of colors perceptible by normal human vision (Chapanis 1954) are reduced to roughly 40 discernible shades of gray. Thus, atmospheric features such as clouds, dust storms, volcanic ash, and smoke/pollution are confined to a very limited grayscale range instead of the more familiar and readily distinguishable whites, yellows, dark browns, and bluish grays seen in true color. While it is often possible to distinguish these various features through crafting of multispectral algorithms displayed as false-color imagery enhancements (e.g., Miller et al. 2006a), mastering the interpretation of such imagery often requires dedicated training and time in handson experience. True color appeals to our "intuitive interpretational skillset" in this regard-our inherent familiarity with the color space of the world as we perceive it with our own eyes.

Despite the clear appreciation and seemingly insatiable appetite for high-quality and visually intuitive satellite imagery among the circles of Earth science research, public/private weather forecasting sectors, and the general public alike, we have operated for the past 50 years without a requirement for true-color imagery on our geostationary (GEO) "imaging” sensors. Not since the very dawn of the meteorological satellite era, for a brief stint on NASA's Applications Technology Satellite 3 (ATS-3) satellite in 1967 (Suomi and Parent 1968; Warnecke and Sunderlin 1968), has a GEO-based sensor offered such a capability. Given the realities of limited satellite program budgets, technical considerations related to focal plane array size, filter technology, and spatial/spectral resolution versus data transmission rate tradeoffs have driven careful prioritization of sensor requirements. Here, true color has been relegated to more of a luxury item rather than a mission-critical necessity.

Compelling arguments for true color do exist, including statements from the founding fathers of satellite meteorology. Suomi and Parent (1968) and Warnecke and Sunderlin (1968) point out some of the unique benefits and information content of the first true-color picture of Earth collected by ATS-3, contrasting it to the conventional grayscale panchromatic visible imagery also available at the time:

This picture is not only a new technology triumph but it reveals a large amount of meteorological information and demonstrates a tremendous potential for meteorological research and operations. A striking advantage over black-and-white photographs is the improved contrast of clouds against the background and the resulting higher effective resolution. Particularly over bright areas with a high surface albedo, color photography obviously permits a better and more detailed detection of cloud distribution and structure.

[...] The superiority of color photography is based on the ability to detect the color peak of the reflectance curve of the different natural objects like water, land, and clouds in addition to the integrated intensity, which is the only parameter detectable by blackand-white photography.

True color has existed for several decades on nonoperational low-Earth-orbiting (LEO) satellite platforms, with more widespread forecast community usage emerging with the launch of NASA's Terra (1999) and Aqua (2002) polar-orbiting satellites [carrying the Moderate Resolution Imaging Spectroradiometer (MODIS)]. The National Oceanic and Atmospheric Administration (NOAA) Joint Polar Satellite System (JPSS), initiated by the Suomi National Polar-Orbiting Partnership (Suomi-NPP) introduced true-color capabilities to the operational program in late 2011 via the Visible Infrared Imaging Radiometer Suite (VIIRS). Global data from these LEO satellites have been made available to users in near-real time and typically provide superior spatial resolution to GEO observations.

There are distinct advantages and limitations to the LEO constellation in terms of coverage and refresh rate. The operational LEO programs, which typically fly in sun-synchronous orbits, provide 90-100-min temporal refresh (with some longer gaps interspersed) at high latitudes. This frequency enables numerous applications that are either unavailable or of superior quality to GEO. However, with these 
same LEO satellites providing only about two local overpasses per 24 -h period at mid- and low latitudes, opportunities to characterize the rapidly changing atmospheric state in these regions are more limited.

At these lower latitudes, the GEO platform takes on added importance. GEO imagery, having the inherent ability to provide rapid refresh rates (looping of imagery to discern motion and evolution of various parameters), is extremely valuable to operational forecasters. Several researchers have demonstrated the advantages to forecasters of high-temporal-resolution imagery and derived products (e.g., Purdom 1976; Schmit et al. 2013; Bedka et al. 2015; Schmit et al. 2015). Here, the regular sampling interval and fixed viewing geometry of the GEO platform complements and augments the detailed information provided by LEO satellites.

The satellite community took "one giant leap for imagery-kind" with the launch of the Japan Meteorological Agency's (JMA) Himawari-8 on 7 October 2014. Himawari (Japanese for "sunflower") is the first of the next generation of GEO satellites, with forthcoming members of this international constellation over the next decade to include NOAA's Geostationary Operational Environmental Satellite R-Series [(GOES-R), carrying the Advanced Baseline Imager (ABI), e.g., Schmit et al. (2005)], the Korea Meteorological Administration (KMA) Geostationary Korea Multipurpose Satellite 2A [(Geo-KOMPSAT$2 A$ ), carrying the Advanced Meteorological Imager (AMI)], the China Meteorological Administration (CMA) Feng Yun-4 [(FY-4), carrying the Advanced Geosynchronous Radiation Imager (AGRI)], and the European Organization for the Exploitation of Meteorological Satellites (EUMETSAT) Meteosat Third Generation [(MTG), carrying the Flexible Combined Imager (FCI)]. The 16-band Advanced Himawari Imager (AHI; built by ITT Exelis, now a part of Harris Corporation) on Himawari-8 features many of the spectral imaging capabilities traditionally relegated to the LEO platform. Thus, AHI represents an important initial test bed for development of applications that can be passed along to other members of the new global constellation.

Here we summarize early developments and present initial results for atmospherically corrected true-color imagery from Himawari-8 AHI. In particular, we detail an adjustment made to AHI's $0.51-\mu \mathrm{m}$ native "green" band that enhances the ability to identify green vegetation and provides a more consistent appearance with respect to legacy polar-orbiting true-color imagery products.

\section{THE HIMAWARI-8 ADVANCED HIMAWARI}

IMAGER. JMA commissioned the Himawari-8 GEO satellite to operational status on 7 July 2015 (Bessho et al. 2016). The satellite subpoint for Himawari-8 is $140.7^{\circ} \mathrm{E}$, hovering over the equatorial region just north of Papua New Guinea and providing a wide ranging view of the western Pacific. The AHI is a state-of-the-art optical spectrum radiometer that acquires multispectral images at high frequency: 10 -min sampling of full-disk imagery and $2.5 \mathrm{~min}$ for regional imagery (collected over Japan and other ephemeral targets of high interest to forecasters, such as typhoons or volcanic eruptions). It includes 3 visible-spectrum (VIS) bands, 3 near-infrared (NIR) bands, and 10 thermal-infrared (IR) bands. The spatial resolutions of these bands are approximately 4 times improved over their counterparts on JMA's heritage Multifunctional Transport Satellites (MTSAT) series imagers.

For interests of this work, we begin by considering the VIS and NIR bands at wavelengths below $1 \mu \mathrm{m}$. Table 1 compares response functions and spatial resolutions of the heritage LEO (MODIS and VIIRS) and next-generation GEO (AHI and ABI) sensors for specific bands of focus. While the red-and blue-band

\begin{tabular}{|c|c|c|c|c|c|c|c|c|c|c|c|c|}
\hline \multirow[t]{2}{*}{$\begin{array}{l}\text { Band } \\
\text { name }\end{array}$} & \multicolumn{3}{|c|}{ MODIS } & \multicolumn{3}{|c|}{ VIIRS } & \multicolumn{3}{|c|}{ AHI } & \multicolumn{3}{|c|}{ ABI } \\
\hline & No. & $\mathrm{FWHM}(\mu \mathrm{m})$ & $\begin{array}{l}\text { Res } \\
(\mathrm{km})\end{array}$ & No. & $\operatorname{FWHM}(\mu \mathrm{m})$ & $\begin{array}{l}\text { Res } \\
(\mathrm{km})\end{array}$ & No. & $\mathrm{FWHM}(\mu \mathrm{m})$ & $\begin{array}{l}\text { Res } \\
(\mathrm{km})\end{array}$ & No. & $\operatorname{FWHM}(\mu \mathrm{m})$ & $\begin{array}{l}\text { Res } \\
(\mathrm{km})\end{array}$ \\
\hline Blue & 3 & $0.459-0.479$ & 0.5 & M3 & $0.478-0.488$ & 0.75 & 1 & $0.43-0.48$ & 1.0 & 1 & $0.45-0.49$ & 1.0 \\
\hline Green & 4 & $0.545-0.565$ & 0.5 & M4 & $0.545-0.565$ & 0.75 & 2 & $0.50-0.52$ & 1.0 & - & - & - \\
\hline Red & 1 & $0.620-0.670$ & 0.25 & M5 & $0.662-0.682$ & 0.75 & 3 & $0.63-0.66$ & 0.5 & 2 & $0.59-0.69$ & 0.5 \\
\hline NIR & 2 & $0.84 I-0.876$ & 0.25 & M7 & $0.846-0.885$ & 0.75 & 4 & $0.85-0.87$ & 1.0 & 3 & $0.846-0.885$ & 1.0 \\
\hline
\end{tabular}




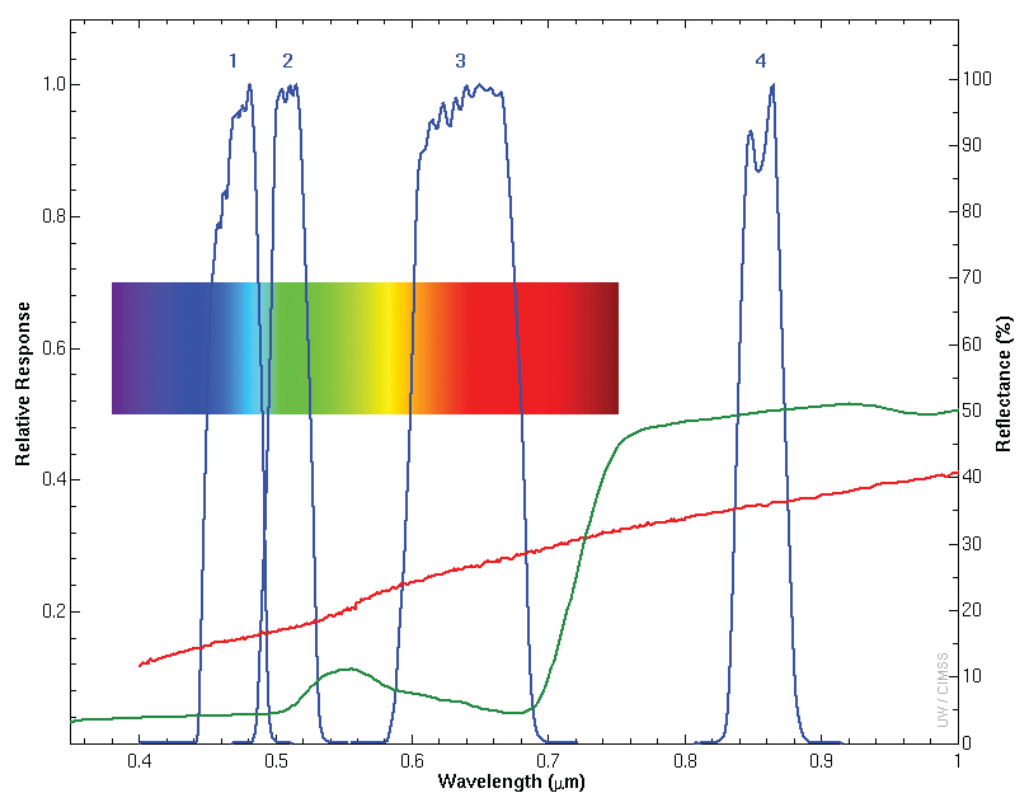

FIG. I. Response functions for the first four AHI bands, shown along with the visible rainbow for reference and green grass vegetation and red-brown sandy loam surface reflectance from the ASTER spectral database.

response functions show minor differences between MODIS/VIIRS and AHI, the green is the only band without overlap-an important point that we shall revisit later. Figure 1 shows the spectral response functions for the sub-1- $\mu \mathrm{m}$ bands of AHI, providing the blue-green-red bands used for true-color imagery as well as the $0.86-\mu \mathrm{m}$ band (often referred to as the "vegetation band" for its strong sensitivity to chlorophyll content, and hence its principal use in monitoring vegetation health). For reference, reflectance data for healthy grass and barren/desert sand taken from NASA's Advanced Spaceborne Thermal Emission and Reflection (ASTER) spectral database are also shown (Baldridge et al. 2009).

TRUE-COLOR PROCESSING WITH RAYLEIGH CORRECTION. Rendering highquality true-color imagery from AHI entails preprocessing of the blue, green, and red (AHI bands 1, 2, and 3) VIS reflectance data for reduction of scattering contributions from the molecular (or Rayleigh; e.g., Young 1981) atmosphere. As Rayleigh scattering efficiency is nonlinearly proportional to inverse wavelength (i.e., $Q_{S} \sim \lambda^{-4}$ ), the atmospheric component of the signal is considerably more noticeable for shorter wavelengths (on AHI, most notable in the $0.47-\mu \mathrm{m}$ blue band). Without reduction of this signal, true-color imagery will take on a milky blue appearance, particularly near the horizon where the viewing path through the atmosphere is long. These adjustments are referred to hereafter as atmospheric "corrections," done to produce a crisper/cleaner version of true-color imagery.

For AHI Rayleigh corrections, we have modified the software of NASA SeaDAS (http://seadas.gsfc .nasa.gov/), a radiative transfer modeling package designed initially for ocean color retrievals. There is no account in the current approximation for aerosol or spatial variations in atmospheric species and pressure. The changes entailed modifying atmospheric absorption parameters, updating the band-integrated solar irradiance, and computing new lookup tables of view and illumination geometry-dependent Rayleigh scattering reflectance corresponding to AHI spectral bands 1-4. The scattering corrections require knowledge of solar and satellite zenith and relative azimuth angles at each pixel. These values are precalculated as a function of image collection date and time. The Rayleigh component is subtracted from the observed reflectance, and this difference is normalized by the atmospheric transmittance for the sun-Earth-satellite photon path. The Rayleighcorrected reflectance values are then scaled log linearly to mimic the nonlinear response of human vision and generally brighten the true-color imagery.

There were some additional adjustments needed to accommodate challenges specific to full-disk imagery. The first dealt with the model's assumption that the atmospheric path always reaches the surface. To avoid overcorrection of pixels at high satellite zenith angles, where high/thick clouds significantly reduce the photon optical path through the atmosphere compared to clear sky, the Rayleigh component was reduced by a factor scaling with cloud-top height (here, using cold AHI 10.35- $\mu \mathrm{m}$ brightness temperatures as a proxy for high clouds). Second, as the correction breaks down nonlinearly at very long atmospheric pathlengths near Earth's limb, uncorrected reflectance data are blended in gradually from satellite zenith angles of $75^{\circ}-85^{\circ}$, carrying on as uncorrected out to the edge of limb. These measures are taken for cosmetic purposes, and they affect a small fraction of the full-disk imagery.

Figure 2 shows uncorrected and atmospherically corrected full-disk AHI true-color imagery for 0230 UTC 25 January 2015-working in this case 
on the first AHI test data made publicly available by JMA. The impact of the atmospheric correction on image sharpness and the general ability to discern land surface features is readily apparent. Optimized coding has enabled end-to-end production of fulldisk imagery in roughly 7 min (using a desktop computer running Linux, equipped with $24 \mathrm{~GB}$ of RAM and a 6-core Intel Xeon W3690 CPU), meaning that 10-min AHI full-disk true-color imagery can currently be processed in real time. Smaller domains require a commensurately smaller fraction of time, such that processing of storm-scale rapid scan sectors should remain computationally tractable.

ANALYSIS REVEALS AN APPARENT ISSUE WITH THE NATIVE AHI GREEN BAND.

Upon closer inspection of Fig. 2b, it becomes evident that the visual appearance of certain land surfaces deviates from the true-color imagery produced by legacy sensors on the LEO satellites mentioned above. Figure $2 \mathrm{c}$ shows the MODIS Blue Marble dataset matched approximately to AHI coverage-taken here as the qualitative standard for expected cloud-free true-color appearance. Comparing Figs. $2 \mathrm{~b}$ and $2 \mathrm{c}$, we notice that vegetation over the tropical Maritime Continent appears "too brown," while the Gobi desert and some parts of the Australian Outback are "too red.” The deviations are explained in both cases by suppression of the green-band component in the RGB composite. Given that AHI's green band was designed to capture green signals explicitly in support of a native true-color imaging capability-the disparity warrants closer inspection.

After confirming that no obvious errors resided in the atmospheric correction code, we turned our attention to the spectral response function of the AHI green band. Figure 3 hones in on the AHI greenband segment of Fig. 2, showing its placement in comparison to the VIIRS moderate-resolution band 4, a $0.55-\mu \mathrm{m}$ band used in Suomi-NPP true-color imagery, and similar to Terra/Aqua MODIS band 4. Also overlaid on Fig. 3 is the chlorophyll signal of healthy green vegetation (extracted from the ASTER spectral library). It is readily apparent that AHI's

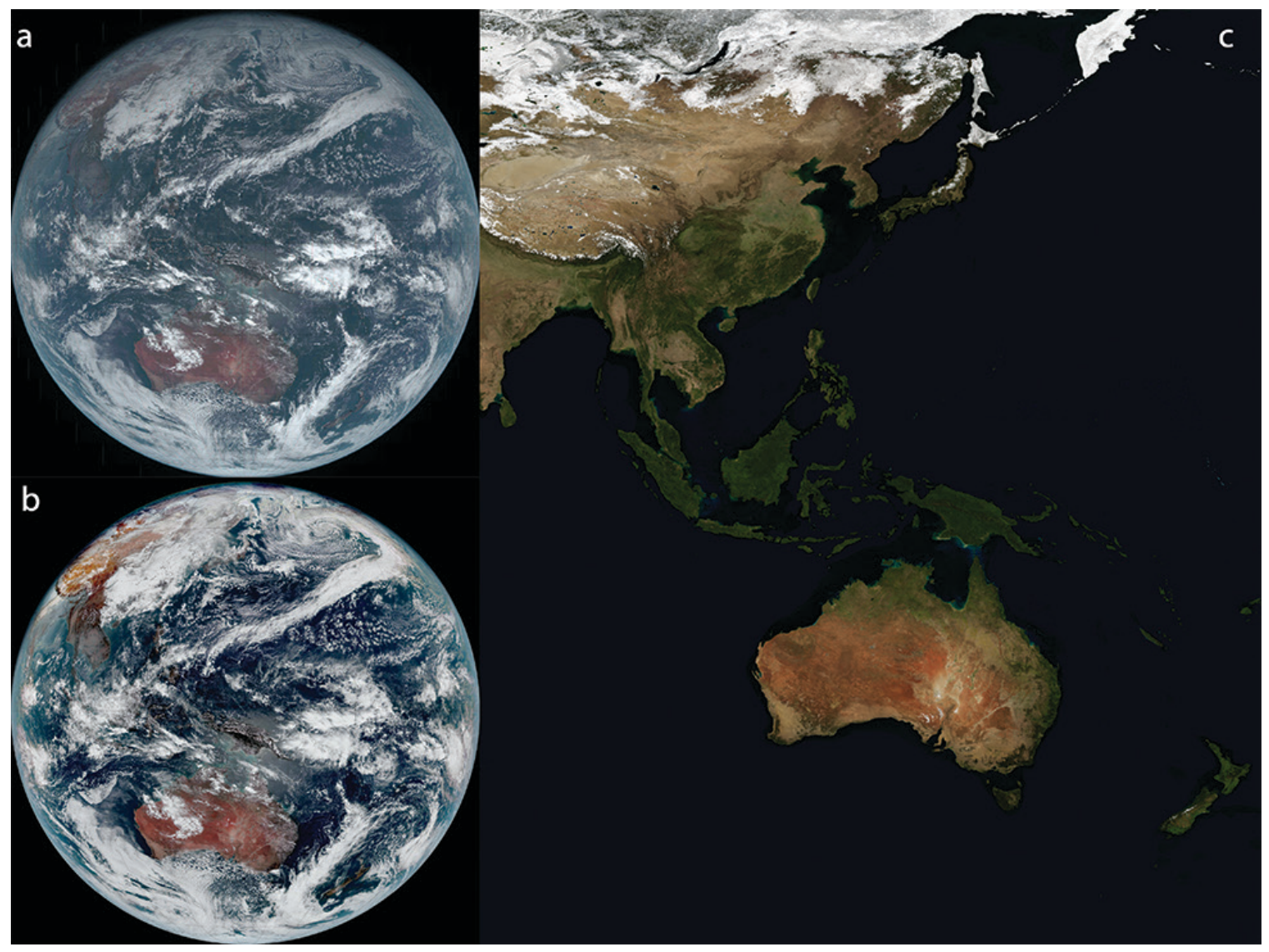

FIG. 2. Example of AHI full-disk true-color imagery (0230 UTC 25 Jan 20I5) (a) without atmospheric correction, (b) with corrections applied, and a subset of MODIS Blue Marble dataset for a domain containing much of AHI's field of regard. 


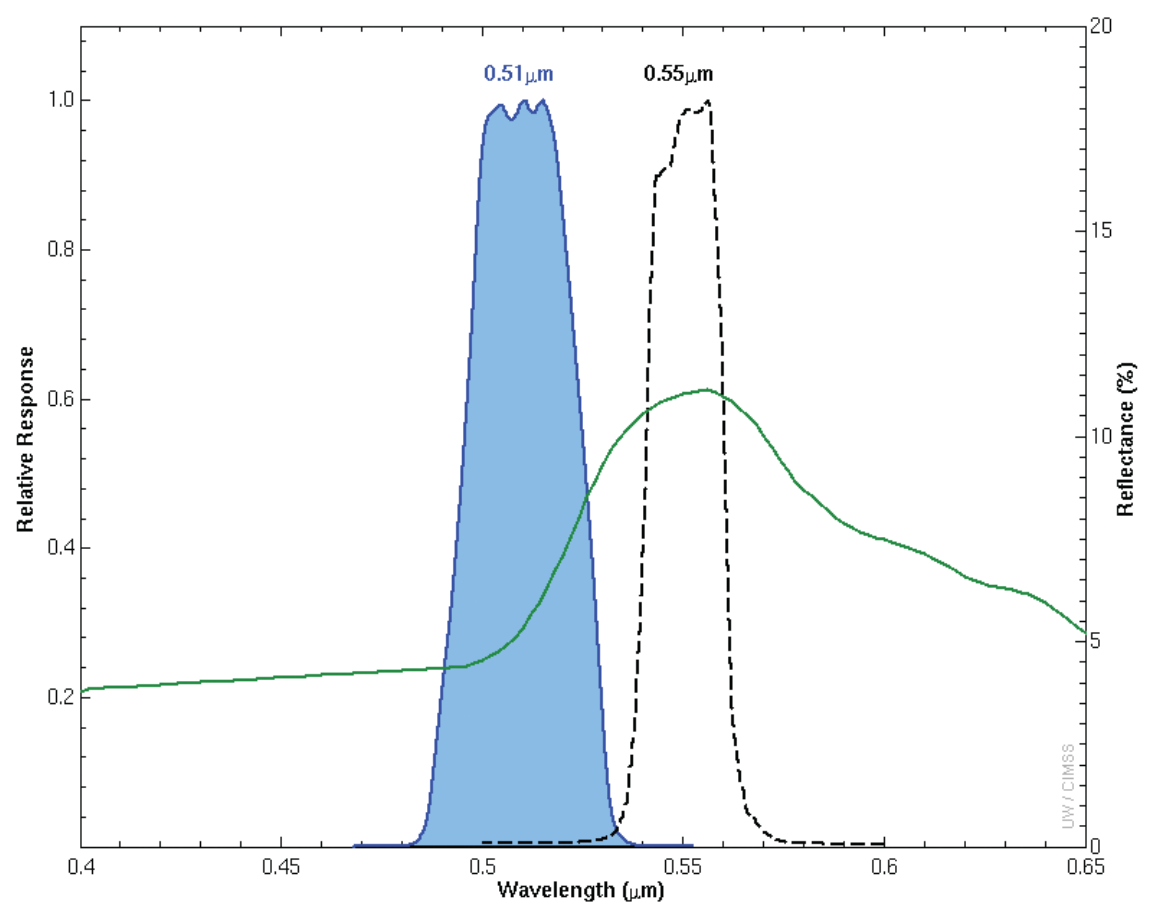

FIG. 3. Zoom in of the AHI green band (blue line and shaded) vs that of SuomiNPP VIIRS (black dashed line), along with grass reflectance (green solid line) from the ASTER spectral database.

green band, while in the green part of the spectrum, is not aligned optimally with the local peak of a typical VIS-spectrum green vegetation reflectance signal. The relative blue shift explains both the suppression of green vegetation and the appearance of redder barren soils (owing to strong absorption by silicates; e.g., Patterson et al. 1977) when comparing AHI true color to the MODIS Blue Marble. Whereas the redand blue-band response functions also differ slightly between MODIS/VIIRS and AHI, unlike the green band they do overlap (Table 1). More importantly, there are not strong, localized, and nonmonotonic spectral variations of surface properties in the blue and red that would be accentuated by differences in the response functions, as seen with chlorophyll signal in the green band.

At this point, it is worth mentioning that by definition true-color images use information only from the current observations and do not rely on ancillary base maps for depicting the land-ocean surface background. Given the lack of the requisite spectral bands for true-color imagery, one approach to approximate true color using data from the current GEO imagers is to use a color base map (sometimes produced from MODIS Blue Marble data; e.g., Miller et al. 2006b) and then overlay the currently observed clouds. While colorful, this approach will not represent as well any short-term surface changes, such as burn scars, vegetation, and water turbidity, that were not present at the time of the base-map compilation. Additionally, subtle atmospheric features, such as thin smoke and cirrus, may be missed more often when merging the current imagery with the base maps if thresholds are not selected carefully and dynamically.

\section{A HYBRID GREEN} SOLUTION. Based on the above analysis, addressing the deficiencies of the native AHI green band would require a nonuniform enhancement of the green signal over certain scene types. A uniform boost to the green reflectance would impart an unrealistic green tint to the imagery, particularly noticeable in the cloud field. Fortunately, a mitigating solution exists within the native AHI spectral suite. Inspecting AHI's $0.86-\mu \mathrm{m}$ vegetation-band spectral response function, shown in Fig. 1, we note that both desert and vegetation reflectance are high compared to the AHI green band. True to the band's nickname, the vegetation signal increases from roughly $5 \%$ to $50 \%$ (strong chlorophyll-A reflectance), but sandy/loam soils' signal increases as well-from about $15 \%$ to $35 \%$ (reduced silicate absorption). Clouds scatter uniformly across the VIS/NIR region, while water bodies are strongly absorbing. As such, AHI band $4(0.86 \mu \mathrm{m})$ provides a boost to signals in exactly those areas where band $2(0.51 \mu \mathrm{m})$ is green deficient, while maintaining a consistent signal in other areas.

With these considerations in mind, we proposed a modification to the AHI native green bandintroducing a small fraction of the AHI vegetationband reflectance to form a hybrid green band that "boosts" the signal selectively over green vegetation and barren desert surfaces. The fractional contribution $F$, which assumes a fixed value between 0 and 1, blends the AHI green and vegetation bands in the following way:

$$
\begin{array}{r}
\text { hybrid green }=(1-F) \times R(0.51 \mu \mathrm{m}) \\
+F \times R(0.86 \mu \mathrm{m}),
\end{array}
$$




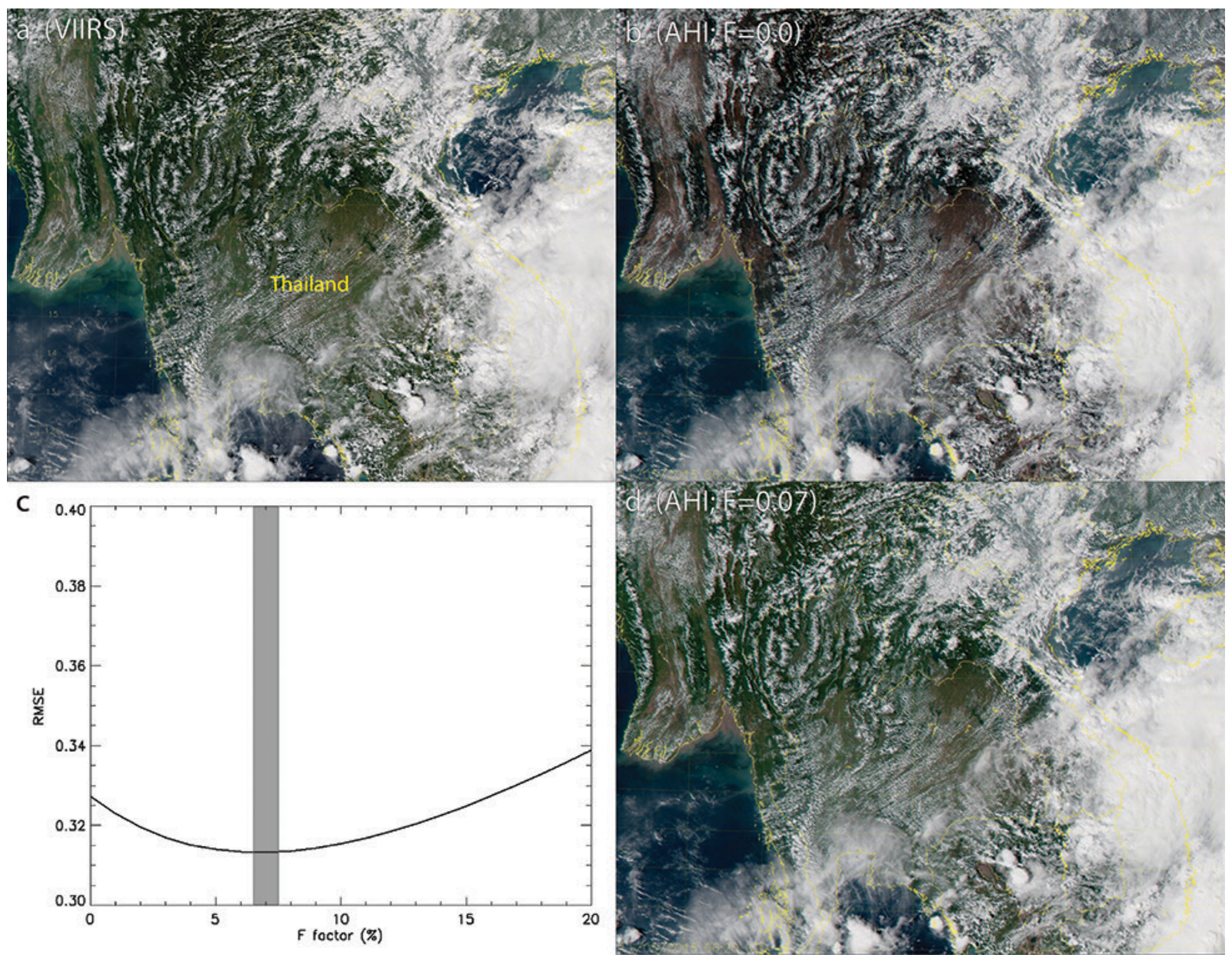

Fig. 4. Comparison of true color imagery collected on 5 November 2015 over the Thailand region for (a) Suomi-NPP VIIRS (06I7 UTC), (b) AHI native band reflectance (0620 UTC), (c) the RMSE minimization to determine hybrid green blending factor $F$, and (d) AHI with hybrid green version of this same scene using a 7\% blend of the AHI $0.86 \mu \mathrm{m}$ vegetation band.

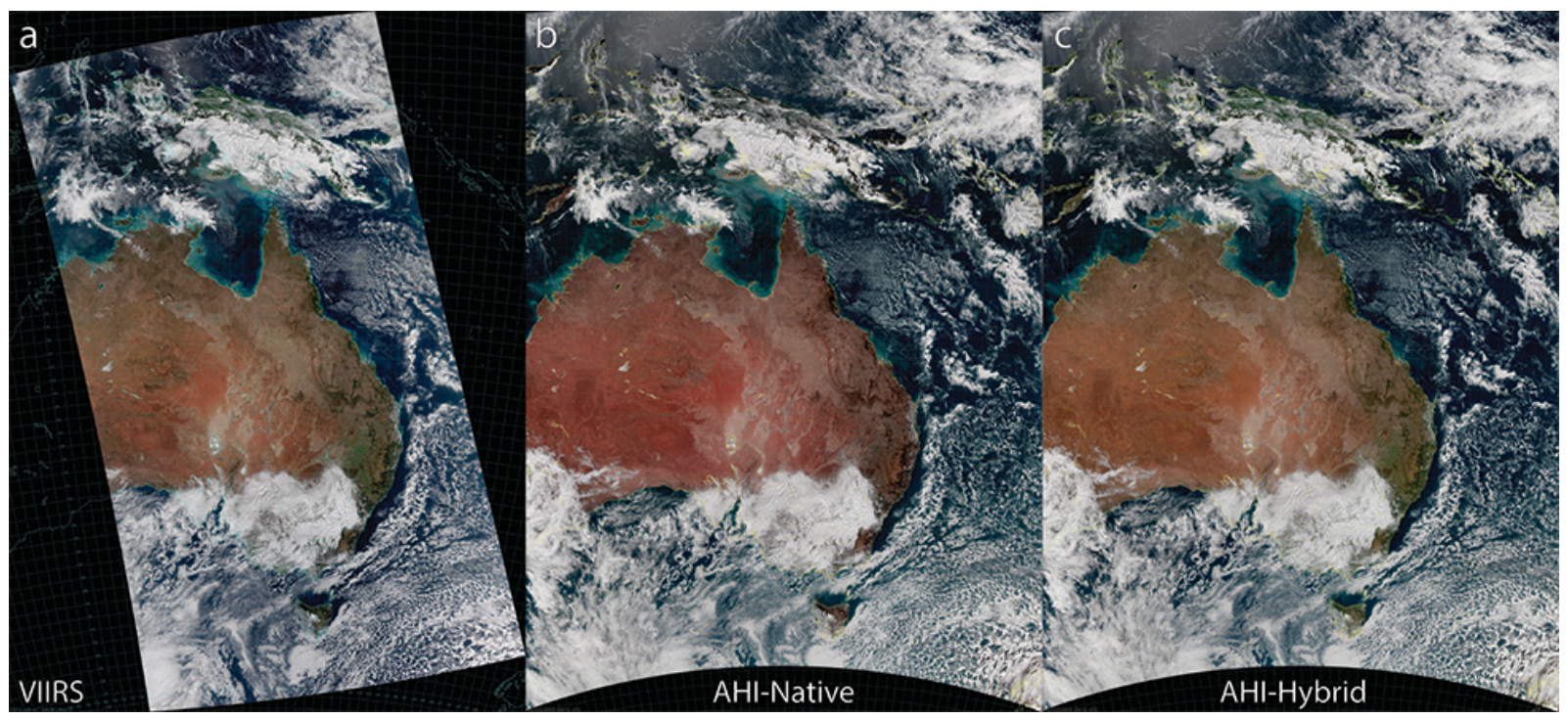

FIG. 5. Comparison of true-color imagery collected on 7 Aug 2015 over the Australian region for (a) SuomiNPP VIIRS (0408 UTC), (b) AHI native-band reflectance matched to this same scene (04I0 UTC), and (c) corresponding $\mathrm{AHI}$ hybrid green version using a $7 \%$ blend of the $\mathrm{AHI}$ 0.86- $\mu \mathrm{m}$ vegetation band. 


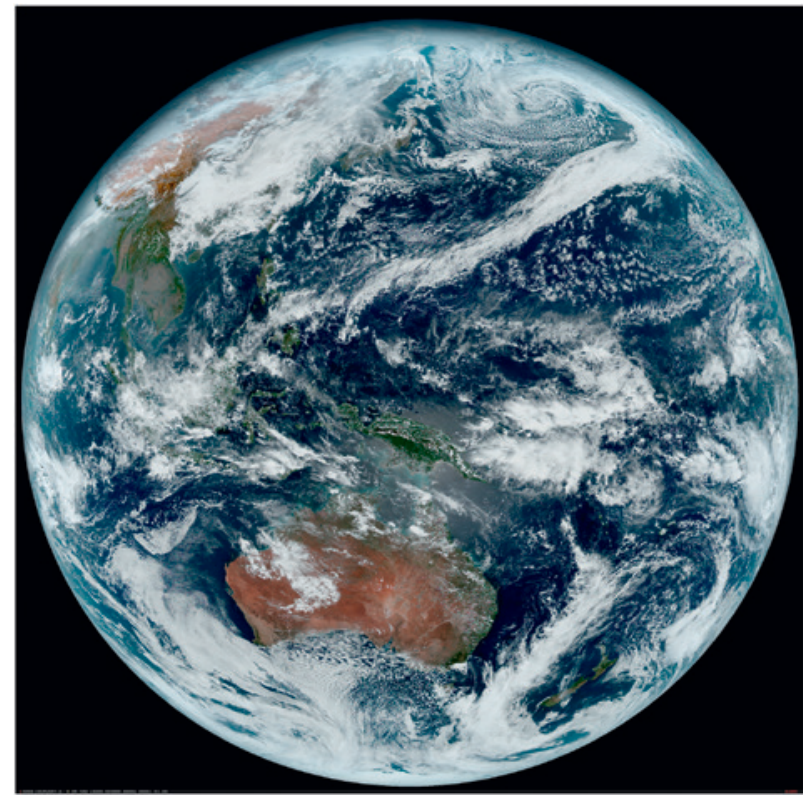

FIG. 6. Final result of processing Himawari-8 AHI truecolor imagery for the same scene as in Fig. 2, with Rayleigh atmospheric corrections and hybrid green [here, F = 0.07; per Eq. (I)] HAC algorithm applied.

where $R$ is the reflectance at the indicated spectral bands.

By definition, the bounds of $F$ preserve either the native AHI green band $(F=0.0)$ or the vegetation band $(F=1.0)$, while values in between yield a blend between the two. Selection of an optimal $F$ was done experimentally, using VIIRS M4 band $(0.555 \mu \mathrm{m})$ as a benchmark. Using space-time matched VIIRS imagery, we varied $F$ until the root-mean-square error (RMSE) between the AHI hybrid green and the VIIRS green reflectance data (both Rayleigh corrected) was minimized. This exercise converged upon a blend factor of $F=0.07$, or roughly a $7 \%$ incorporation of the $0.86-\mu \mathrm{m}$ band, for optimal matching with VIIRS. Figure 4 shows the results for an example over Thailand. Given the strong response of the vegetation band, only a small contribution is necessary to achieve an acceptable result. As with any enhancement, selection of $F$ can be tailored to suit specific user needs. At full-disk imagery scale, slightly higher values of $F$ (up to about 0.15 ) can be used to further accentuate vegetation-dense regions. However, it was found that values higher than $F \approx 0.20$ produced large RMSE and the anomalous greening of deserts.

Figure 5 shows another comparison between AHI (uncorrected green band in Fig. 5a, hybrid green in Fig. 5b) and VIIRS (Fig. 5c) for closely matched, Rayleigh-corrected observations over Australia, using $F=0.07$. Notable areas of increased green vegetation over Indonesia, Papua New Guinea, and parts of northern and eastern Australia improve the visual agreement with the VIIRS reference imagery. The crossover of vegetation and sand reflectance between 0.51 and $0.86 \mu \mathrm{m}$ (Fig. 1) suggests that a spatially invariant blend factor $F$ in Eq. (1) will necessarily impart a stronger enhancement to vegetation than to deserts. Figure 5 suggests that the visual effects of this disproportionate adjustment are slight, with the benefits outweighing the physical limitations of this simple approach. It is also evident that coastal waters, which may contain shades of turquoise/green depending on bathymetry (shallow waters) and turbidity (northern coast of Australia in Fig. 5), are not affected significantly by the hybrid green technique. For these scene types, their green tonality arises from the spectral mixing of broad reflectance signatures rather than a narrowband increase in green reflectance near $0.55 \mu \mathrm{m}$ due to chlorophyll-A. This is why these coastal waters still appear as green/turquoise, and not brown like the vegetation, in the native AHI true-color imagery of Fig. 5a. While the agreement is not perfect, the hybrid approach appears to capture to first order the appearance of legacy true-color products.

Figure 6 shows the end product of the AHI processing detailed above. We refer to this approach as "hybrid, atmospherically corrected" (HAC) true color. As mentioned previously, a slight blend of uncorrected AHI data are introduced near Earth's limb to provide better consistency with respect to the MODIS and VIIRS Blue Marble imagery, while also covering exponentially growing errors in the Rayleigh correction at long optical paths through the atmosphere. With Rayleigh correction and a dynamic boost applied to the green band, AHI's captures our planet in the same colorful splendor as versions composited from polar-orbiting satellite sensors, but for the first time as a single contiguous image with an ability to animate at 10 -min intervals over the full disk, and as often as every 2.5 min over target areas. When animated at the scale of full-disk imagery, the cloud and aerosol patterns (now readily distinguishable via true color) appear to evolve smoothly at this temporal resolution.

\section{A SIMPLE HYBRID, CONTRAST-STRETCH} TECHNIQUE. The simple hybrid contrast-stretch (SHCS) approach is a middle-ground solution for preparing true-color imagery that is "cleaner" than a direct combination of the red-green-blue AHI bands, but without incurring the computational burden of atmospheric corrections required by the 
HAC approach. Here, each of the color components is stretched to suppress the atmospheric signal that HAC attempts to remove explicitly. The uncorrected reflectance data $\left(\mathrm{R}_{\text {orig; }} ; \%\right)$ are first expressed in terms of 8-bit integer scaling, where 0 represents the minimum reflectance $\left(\mathrm{R}_{\min }\right)$ and 255 represents the maximum reflectance $\left(\mathrm{R}_{\max }\right)$ of the scaling bounds. For the current examples, we have set $\left[R_{\min }, R_{\max }\right]$ to $[0,125] \%$ for all three (red, hybrid green, and blue) AHI bands. Values higher than 100\% on the upper bounds of this range accommodate three-dimensional effects such as the side illumination of clouds. The following approach is used to conduct this 8-bit integer scaling:

$$
\begin{gathered}
\mathrm{R} \_ \text {new }=255 \times\left(\mathrm{R}_{\text {orig }}-\mathrm{R}_{\min }\right) \\
/\left(\mathrm{R}_{\max }-\mathrm{R}_{\min }\right) .
\end{gathered}
$$

These scaled reflectance are then rescaled piecewise linearly over three predefined ranges, shown in Table 2. This rescaling follows the same general construct as Eq. (2) (but without the 255 premultiplier).
For SHCS, the AHI hybrid green band uses $F=0.13$ to attain optimal color matching with the legacy MODIS/VIIRS green band. The rescaled bands are then combined to form the SHCS true-color imagery. The minimum values used for restretching were determined empirically, based on a relatively small set of sample data, and therefore may require fine tuning as a function of season, location, and time of day.

The net effect of the SHCS rescaling is to increase the dynamic range of the midrange reflectance values at the expense of the low- and high-end ranges. The method achieves a pseudo-Rayleigh correction by applying successively stronger low-end

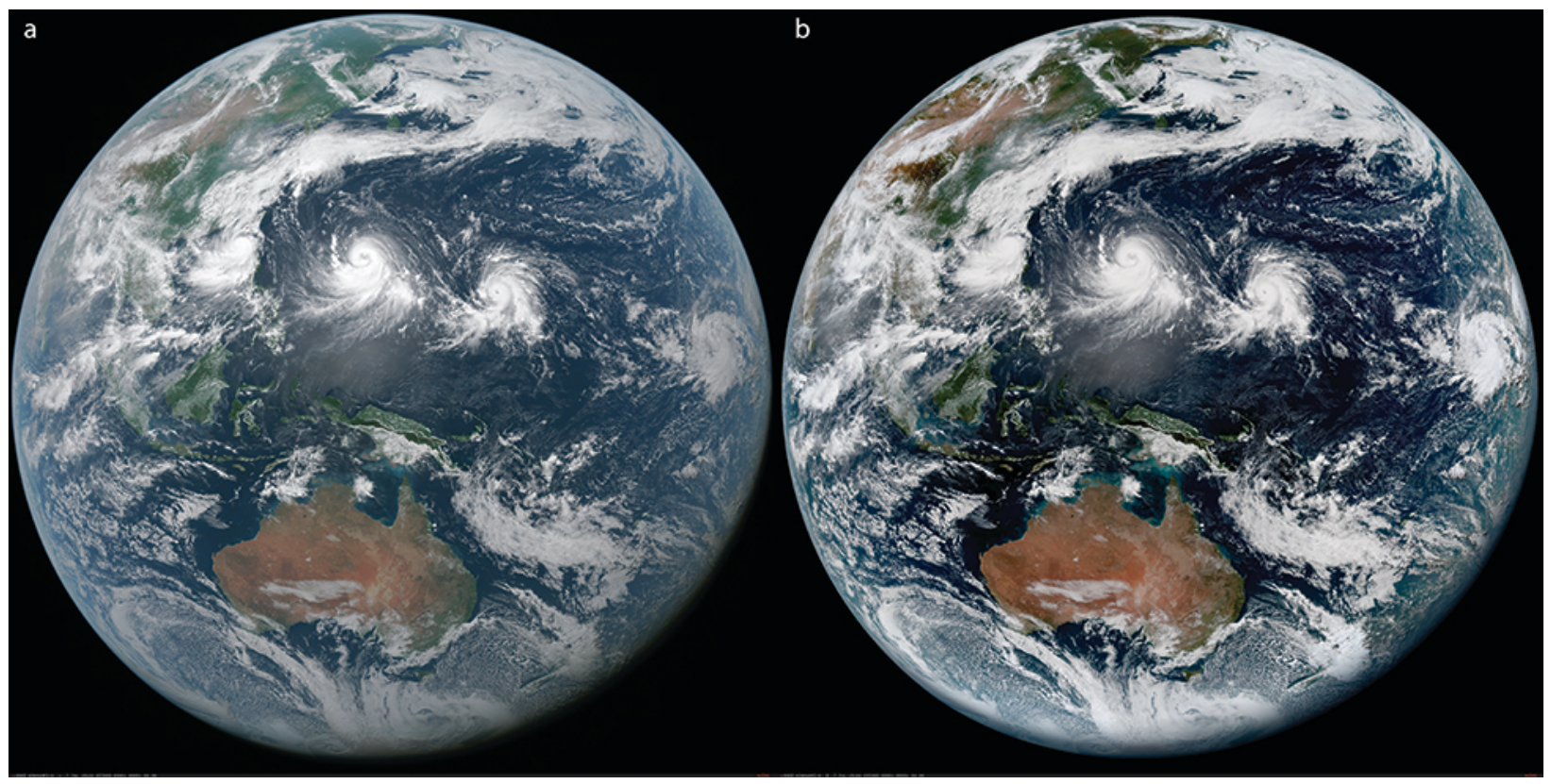

FIG. 7. Comparison of AHI true-color imagery based on (a) the SHCS method $[F=0.13$; per Eq. (I)] and (b) the HAC method $(F=0.07)$ for full-disk imagery collected at 0330 UTC 7 Jul 2015. A string of three tropical storms seen churning over the western Pacific Ocean are, from approximate image center to eastern limb, Typhoon Chan-hom, Typhoon Nangka, and Tropical Storm Linfa. Animations of this example for uncorrected, SHCS, and HAC true-color imagery are available at http://dx.doi.org/0.1175/BAMS-D-15-00154.2. 
suppressions for the green and blue bands. Figure 7 compares the SHCS approach against the fully corrected (HAC) AHI imagery for full-disk AHI imagery, collected at 0330 UTC 7 July 2015 . This particular date holds the distinction of being the official operational commissioning of Himawari-8. A video of the HAC method for this date is included in the online supplement for this article (available online

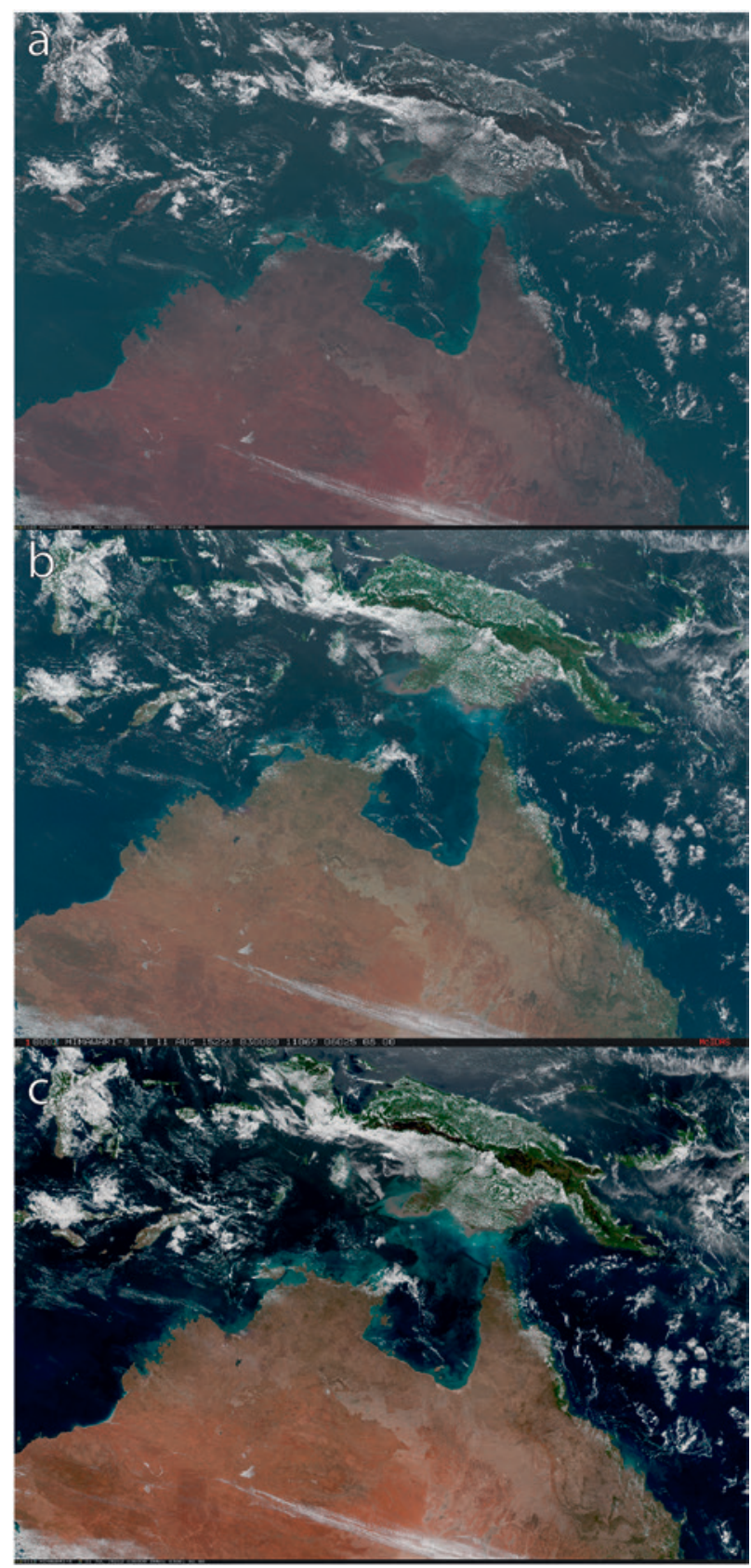

FIG. 8. Comparison of AHI true-color imagery for (a) native $0.5 \mathrm{I}-\mu \mathrm{m}$ green band and no atmospheric correction, (b) SHCS method [with $F=0.13$; per Eq. (I)], and (c) HAC method (with $F=0.07$ ) for a subset of imagery over northern Australia at 0300 UTC II Aug 2015. at http://dx.doi.org/I0.II75/BAMS-D-I5-00I54.2). Figure 8 hones in on a comparison of unaltered reflectance, SHCS and HAC true-color imagery for a zoomed-in region of northern Australia at 0300 UTC 11 August 2015. Both methods provide a visually superior result to native-band imagery through the suppression (either via scaling in the case of SHCS, or physical account via HAC) of the Rayleigh signal. When comparing the two methods, the main advantage of the SHCS approach is that it is very fast to process, having no Rayleigh corrections or attendant requirements for solar/satellite geometry information. An additional benefit of SHCS is that no processing artifacts from Rayleigh corrections are introduced near the terminator. The principal disadvantage is that SHCS will not appear as vivid as the HAC method in some areas and/or at certain times of day, since the molecular signal is in fact still present in all of the bands used. As a result, certain features such as thin smoke, water shoals/turbidity, and phytoplankton blooms may not stand out as readily as they do in HAC true-color imagery, particularly at more oblique view angles. It is possible that a time-dependent scaling of SHCS could improve performance, and this is an area of ongoing study.

ILLUSTRATIVE EXAMPLES. The benefits of true color for scene interpretation are best appreciated when honing in on specific examples at higher spatial resolution. Figure 9 highlights several examples of atmospheric aerosol originating from both natural and anthropogenic sources, as seen with AHI's HAC true-color algorithm. Volcanic ash plumes may take on a variety of colors depending on the nature and composition of the ash plume (e.g., Yamanoi et al. 2008). In Fig. 9a, an explosive eruption of the Manam Volcano in Papua New Guinea produced a "mushroom" cloud with tan coloration. The ability to distinguish plumes of different constituents is highlighted in Fig. 9b, where the residual dark smoke from the Tianjin, China, industrial explosion stands out readily against the semipersistent grayish-blue haze of pollution in southeastern China. Looking toward the limb of AHI's field of view, Fig. 9c shows massive smoke plumes billowing from a complex of Russian forest fires burning out of control near Lake Baikal, and Fig. $9 \mathrm{~d}$ captures the advance of an expansive wall of desert dust meandering across the complex terrain of central China.

With the aid of true-color imagery, these varying features appear readily and distinctly from surrounding meteorological clouds. Includ- 
ing the temporal dimension further assists in feature discrimination and interpretation. Videos corresponding to subpanels of Fig. 9 and the full-disk scene of Fig. 7, shown at various stages of true-color imagery enhancement ranging from no adjustments whatsoever (native-band reflectance), to SHCS, to the full HAC approach, are provided as online supplemental information to this article.

DISCUSSION. AHI's green band was selected by JMA for the purpose of providing a native truecolor imaging capability. The obvious question that arises from this discussion is why the band was positioned outside the green peak of the vegetation spectral reflectance spectrum? Personal communications between the authors and representatives of EUMETSAT and JMA may help to shed light on this question. During the scoping of Europe's nextgeneration geostationary satellite imager, the MTG/ FCI, EUMETSAT selected a $0.51-\mu \mathrm{m}$ green band for aerosol property retrievals. This green was not aligned with the $0.55-\mu \mathrm{m}$ chlorophyll-A structure, since its user base had not voiced a specific requirement for true-color imagery at that time. Subsequently, JMA adopted this $0.51-\mu \mathrm{m}$ aerosol-centric green band for Himawari-8 AHI, but with the expectation that it would satisfy their requirements for true-color imagery. The KMA adopted the same EUMETSAT/ JMA green band for Geo-KOMPSAT-2A AMI, also for the purposes of supporting true-color imagery. Whereas the underpinning requirement for the green band changed along the way, the corresponding green-band specification apparently did not.

It is worth noting that in the early design phase of the GOES-R ABI, a $0.55-\mu \mathrm{m}$ band was proposed for the express purpose of supporting true-color imagery. In the design trade space considered for the 16-band sensor, only six reflected-solar bands could fit on the focal plane array. The requirement for subvisual cirrus detection drove selection of the $1.38-\mu \mathrm{m}$

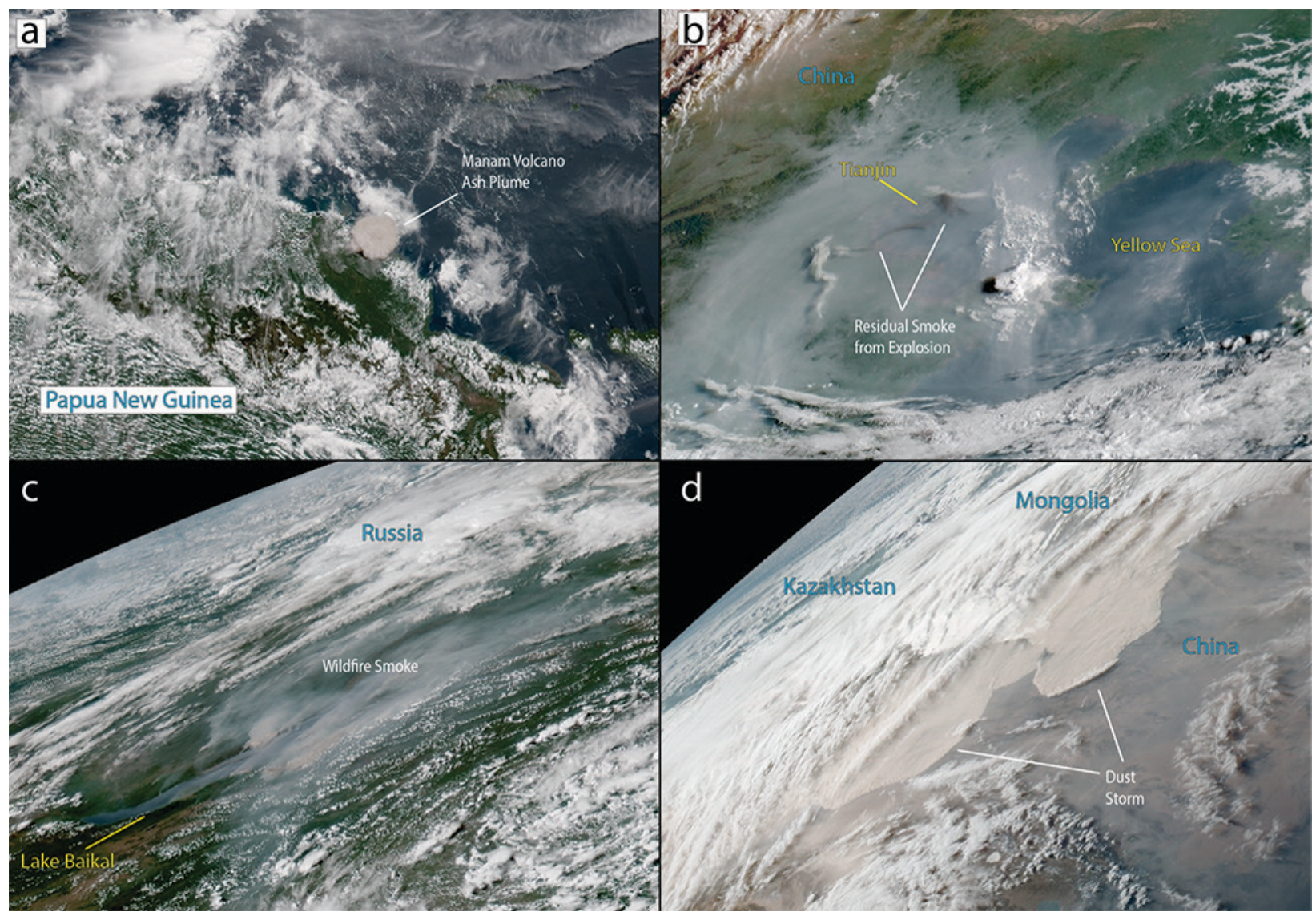

FIG. 9. A variety of atmospheric features whose interpretation is facilitated by HAC true-color AHI imagery: (a) volcanic ash plume from the Manam Volcano in Papua New Guinea (3I Jul 20l5); (b) a dark smoke plume associated with the Tianjin, China, industrial explosions drifts with lighter-gray background pollution (I 2 Aug 20I5); (c) biomass smoke from wildland fires raging near Lake Baikal in south-central Russia (7 Aug 20I5); and (d) desert dust pooling over central China (27 Apr 2015). Animations of these cases for uncorrected, SHCS, and HAC true-color imagery are available at http://dx.doi.org/I0.1I75/BAMS-D-I5-00I54.2. 
(shortwave water vapor) band, and in the case of GOES-R that came at the expense of the green band. Ultimately, true-color imagery lacked a "validated user requirement" (Schmit et al. 2003) strong enough to outrank higher priority requirements. Adding weight to the true-color requirement in the future will take a formal assessment by the international user community, demonstrating how the information content of true-color imagery is of practical value for scene interpretation (including delineation of visibility hazards such as smoke and blowing dust). Quantitative metrics, such as determining an analyst's ability to distinguish smoke, dust, and cloud features using true-color versus standard VIS-NIR-IR imagery in a time-limited setting, or development of objective color-space feature discrimination algorithms, would be useful in this regard. Despite the specific limitations of this new generation of GEO sensors for rendering true-color imagery, it is important to remember that their overall abilities represent a tremendous advance, and we have demonstrated here that via simple techniques the obstacles are at least partially surmountable.

An important lesson learned for the design of future sensors intended for true-color imaging is to center their green band's peak sensitivity, near $0.55 \mu \mathrm{m}$. The Coordination Group for Meteorological Satellites (CGMS) is perhaps the most appropriate purveyor of this best-practices guidance to the international community of meteorological satellite developers. In the meantime, and in the immediacy of next-generation GEO systems coming online, it is likely that the international user community will instead adopt some version of this effective and straightforward hybrid green technique to mitigate the $0.51-\mu \mathrm{m}$ deficiencies when producing true-color imagery for their respective programs.

The situation is a bit more complicated for the U.S. program, but remains manageable. The ABI, which will fly on NOAA's next-generation GOES-R series and was in fact built by the same vendor as AHI, carries 16 spectral bands as well. However, ABI will include a NIR band centered at $1.38 \mu \mathrm{m}$ instead of a green band. The $1.38-\mu \mathrm{m}$ band, not present on AHI, holds great value in its own right, being able to detect daytime cirrus (Gao et al. 2002) owing to its unique spectral location in the center of a strong water vapor absorption band. However, it leaves GOES-R without an immediate means for rendering true-color imagery from its native suite of bands.

In this regard, the current AHI research takes on added significance-preparing the way for advanced techniques that will enlist Himawari-8 AHI as training for a "synthetic" or "virtual" green band, via correlations that exist between green and adjacent VIS/NIR bands (e.g., Miller et al. 2012; Hillger et al. 2011; Gladkova et al. 2011). Since AHI contains blue, red, and vegetation bands common to $\mathrm{ABI}$, along with the requisite green band, it can be used as the superset of spectral information necessary to formulate this relationship. These ABI-preparatory developments represent the next logical pursuits of the current research.

CONCLUSIONS. At long last, Japan's Himawari-8 AHI marks the return of true-color capabilities to the geostationary platform. While first-light imagery revealed a suboptimal green band, through a bit of "spectral smithery," a hybrid technique has been crafted to overcome this band's main deficiencies. A subtle blend of the native $0.51-\mu \mathrm{m}$ green band with the $0.86-\mu \mathrm{m}$ vegetation band produces a selectively boosted green band yielding true-color imagery that is consistent with the appearance of legacy polarorbiting sensors. The HAC correction is by no means a panacea, but in the realm of multispectral imagery enhancement development (which often resorts to a kind of "whack-a-mole" game, wherein the fixing of one issue gives rise to yet another issue, with iterations continuing ad infinitum), the availability of a simple, bispectral solution is serendipitous. It can be regarded as a positive outcome to what might otherwise have meant going without high-quality true-color imagery on the GEO platform.

It is worth noting that NASA's Earth Polychromatic Imaging Camera (EPIC) on the Deep Space Climate Observatory (DSCOVR; Early et al. 2002), launched on 11 February 2015, now provides a unique true-color imagery capability from the Lagrange-1 orbit (L1; 1.6 million $\mathrm{km}$ away from Earth at a gravitationally stable point between Earth and the sun) that views the daylit side of Earth on a nearly continual basis. Since L1 is not by definition a GEO orbit, there is only one view from a given longitudinal perspective per day, provided at roughly $10-\mathrm{km}$ (subsatellite point) resolution, hourly data, and at 12 -h latency. This is an important distinction between the two platforms; the GEO imagers provide 10-15-min full-disk updates at $10 \mathrm{~min}$ latency, with approximately $1-\mathrm{km}$ resolution at subpoint and a fixed viewing perspective. The unique perspective of EPIC holds value for numerous quantitative applications in terms of multiangle viewing (e.g., for cloud/aerosol geometric and optical properties). While the EPIC data are not a viable alternative to scene-fixed, high-space-time-resolution GEO observations, they offer a novel way of viewing Earth 
and a gap filler to true-color imaging from the nascent GEO constellation.

After going without a true-color capability on GEO satellites for nearly 50 years, we suddenly find ourselves in an embarrassment of riches, with true-color-equipped sensors on the LEO, GEO, and L1 orbits. True color plays a far greater role than simply serving as the flagship public relations material for our next-generation GEO programs; it offers the human analyst a practical tool for intuitive scene interpretation. Over the next decade, the full international complement of GEO sensors will begin using true color to reveal the dynamic Earthatmosphere system in an entirely new, yet inherently familiar way. While it may require some fine tuning and processing "HACs" for the time being, true-color imagery is back on GEO, and hopefully this time it is here to stay!

ACKNOWLEDGMENTS. Support of the NOAA GOES-R Program Office, the Naval Research Laboratory (Contract N00173-14-G902), the Oceanographer of the Navy PEO C4I and Space/PMW-120, Program Element PE-0603207N, and the Office of Naval Research (Contract N00014-16-1-2040) are gratefully acknowledged. The views, opinions, and findings in this report are those of the authors and should not be construed as an official NOAA and/or U.S. Government position, policy, or decision. We thank Jean Phillips and Jaclyn Lang of the SSEC Schwerdtfeger Library for providing background material on ATS-3. More information on true-color images can be found with the following search keywords: JMA AHI imagery, CIRA true-color AHI, SSEC geostationary browser AHI, and SSEC Real Earth AHI true color. Real-time AHI imagery can be found at http://rammb.cira.colostate .edu/ramsdis/online/himawari-8.asp. Various blog entries expanding on the capabilities of Himawari-8 can be found at http://cimss.ssec.wisc.edu/goes/blog/archives /category/himawari-8. Finally, a web-based application intended to help users better understand RGB formulation, including the effects of atmospheric correction, can be found at http://cimss.ssec.wisc.edu/goes/webapps/satrgb /satrgb_AHI_color.html.

\section{REFERENCES}

Baldridge, A. M., S. J. Hook, C. I. Grove, and G. Rivera, 2009: The ASTER Spectral Library Version 2.0. Remote Sens. Environ., 113, 711-715, doi:10.1016/j .rse.2008.11.007.

Bedka, K. M., C. Wang, R. Rogers, L. D. Carey, W. Feltz, and J. Kanak, 2015: Examining deep convective cloud evolution using total lightning, WSR-88D, and
GOES-14 super rapid scan datasets. Wea. Forecasting, 30, 571-590, doi:10.1175/WAF-D-14-00062.1.

Bessho, K., and Coauthors, 2016: An introduction to Himawari-8/9-Japan's new-generation geostationary meteorological satellites. J. Meteor. Soc. Japan, 94, 151-183, doi:10.2151/jmsj.2016-009.

Chapanis, A., 1954: Color names for color space. Amer. Sci., 53, 327-346.

d'Entremont, R. P., and L. W. Thomason, 1987: Interpreting meteorological satellite images using a color composite technique. Bull. Amer. Meteor. Soc., 68, 762-768, doi:10.1175/1520-0477(1987)068<0762 :IMSIUA $>2.0 . \mathrm{CO} ; 2$.

Early, E. A., B. C. Bush, S. W. Brown, D. W. Allen, and B. C. Johnson, 2002: Radiometric calibration of the Scripps Earth Polychromatic Imaging Camera. Earth Observing Systems VI, W. L. Barnes, Ed., International Society for Optical Engineering (SPIE Proceedings, Vol. 4483), doi:10.1117/12.453474.

Gao, B.-C., P. Yang, W. Han, R.-R. Li, and W. J. Wiscombe, 2002: An algorithm using visible and $1.38-\mathrm{mm}$ channels to retrieve cirrus cloud reflectances from aircraft and satellite data. IEEE Trans. Geosci. Remote Sens., 40, 1659-1668, doi:10.1109 /TGRS.2002.802454.

Gladkova, I., F. Shahriar, M. Grossberg, G. Bonev, D. Hillger, and S. Miller, 2011: Virtual green band for GOES-R. Earth Observing Systems XVI, J. J. Butler, $\mathrm{X}$. Xiong, and X. Gu, Eds., International Society for Optical Engineering (SPIE Proceedings, Vol. 8153), doi:10.1117/12.893660.

Hillger, D., L. Grasso, S. D. Miller, R. Brummer, and R. DeMaria, 2011: Synthetic GOES-R Advanced Baseline Imager true color imagery. J. Appl. Remote Sens., 5, 053520, doi:10.1117/1.3576112.

Miller, S. D., and Coauthors, 2006a: MODIS provides a satellite focus on Operation Iraqi Freedom. Int. J. Remote Sens., 27, 1285-1296, doi:10.1080 /01431160500383772.

— - and Coauthors, 2006b: NexSat: Previewing NPOESS/VIIRS imagery capabilities. Bull. Amer. Meteor. Soc., 87, 433-446, doi:10.1175/BAMS-87-4-433.

—, C. Schmidt, T. Schmit, and D. Hillger, 2012: A case for natural colour imagery from geostationary satellites, and an approximation for the GOES-R ABI. Int. J. Remote Sens., 33, 3999-4028, doi:10.1080 /01431161.2011.637529.

Patterson, E. M., D. A. Gillette, and B. H. Stockton, 1977: Complex index of refraction between 300 and $700 \mathrm{~nm}$ for Saharan aerosols. J. Geophys. Res., 82, 3153-3160, doi:10.1029/JC082i021p03153.

Purdom, J. F. W., 1976: Some uses of high-resolution GOES imagery in the mesoscale forecasting of 
convection and its behavior. Mon. Wea. Rev., 104, 1474-1483, doi:10.1175/1520-0493(1976)104<1474: SUOHRG $>2.0 . \mathrm{CO} ; 2$.

Schmit, T. J., W. P. Menzel, J. Sieglaff, J. P. Nelson III, M. K. Griffin, and J. J. Gurka, 2003: Channel selection for the next generation geostationary Advanced Baseline Imagers. Preprints, 12th Conf. on Satellite Meteorology and Oceanography, Long Beach, CA, Amer. Meteor. Soc., P5.3. [Available online at https:// ams.confex.com/ams/annual2003/techprogram /paper_54285.htm.]

— , M. M. Gunshor, W. P. Menzel, J. Li, S. Bachmeier, and J. J. Gurka, 2005: Introducing the next-generation advanced baseline imager on GOES-R. Bull. Amer. Meteor. Soc., 86, 1079-1096, doi:10.1175/BAMS-86-8-1079.

—_, and Coauthors, 2013: Geostationary Operational Environmental Satellite (GOES)-14 super rapid scan operations to prepare for GOES-R. J. Appl. Remote Sens., 7, 073462, doi:10.1117/1.JRS.7.073462.
— of significant events: Preparing users for the next generation of geostationary operational satellites. Bull. Amer. Meteor. Soc., 96, 561-576, doi:10.1175 /BAMS-D-13-00210.1.

Suomi, V. E., and R. Parent, 1968: A color view of Planet Earth. Bull. Amer. Meteor. Soc., 49, 74-75.

Warnecke, G., and W. Sunderlin, 1968: The first color picture of the earth taken from the ATS3 satellite. Bull. Amer. Meteor. Soc., 49, 75-83.

Yamanoi, Y., S. Takeuchi, S. Okumur, S. Nakashima, and T. Yokoyama, 2008: Color measurements of volcanic ash deposits from three different styles of summit activity at Sakurajima volcano, Japan: Conduit processes recorded in color of volcanic ash. J. Volcanol. Geotherm. Res., 178, 81-93, doi:10.1016/j .jvolgeores.2007.11.013.

Young, A. T., 1981: Rayleigh scattering. Appl. Opt., 20, 533-535, doi:10.1364/AO.20.000533.

\section{NEW FROM AMS BOOKS!}

\section{"A thoughtful analysis of actions that we need to take to reduce the impacts of extreme weather...a must-read for everyone with an interest in the weather and climate." \\ - FRANKLIN W. NUTTER, \\ President, Reinsurance Association of America}

\section{Living on the Real World: How Thinking and Acting Like Meteorologists Will Help Save the Planet}

WILLIAM H. HOOKE

Meteorologists sift through a deluge of information to make predictions every day. Instead of being overwhelmed by the data and possibilities, they focus on small bits of information while using frequent collaboration to make decisions. With climate change a reality, William H. Hooke suggests we look to the way meteorologists operate as a model for how we can solve the twenty-first century's most urgent environmental problems. 